\title{
Correction to: Homogenization of an elastic thin plate
}

\section{Correction to:}

Chapter 11 in: D. Cioranescu et al., The Periodic Unfolding Method, Series in Contemporary Mathematics 3, https://doi.org/10.1007/978-981-13-3032-2_11

The original version of the chapter was inadvertently published without the proof for Lemma 11.11, which has now been included. The correction chapter has been updated with the change. 